\title{
MELHORAMENTO DA CANA-DE-AÇÚCAR: X. ENSAIO DE CLONES PROVENIENTES DE HIBRIDAÇÕES REALIZADAS EM 1981 E SELECIONADOS NA REGIÃO DE JAÚ (SP) $\left(^{1}\right)$
}

\author{
MÁRIO PERCIO CAMPANA $\left({ }^{2}\right)$, MARCELO DE ALMEIDA SILVA $\left({ }^{2}\right)$, \\ RAPHAEL ALVAREZ $\left({ }^{3}\right)$, MARCOS GUIMARÃES DE ANDRADE LANDELL $\left({ }^{4,6}\right)$, \\ PERY FIGUEIREDO $\left({ }^{5}\right)$ e DENIZART BOLONHEZI $\left({ }^{4}\right)$
}

\begin{abstract}
RESUMO
Avaliaram-se sete clones de cana-de-açúcar provenientes de hibridações realizadas em Camamu (BN), em 1981, em um ensaio instalado na região de Jaú (SP), em fevereiro de 1988. Utilizou-se o delineamento em blocos ao acaso, com seis repetições, no qual se avaliou a produtividade de cana e açúcar e pol\% cana, em média, de cinco colheitas. Apresentam-se, ainda, dados de população de colmos, fibra\% cana $e$ intensidade de florescimento. Em relação às variedades IAC67-112, NA56-79, SP70-1143, SP71-1406 e PO77-102, empregadas como padrão, os clones IAC81-3041 e IAC81-3049 revelaram-se promissores, apresentando condiçōes de ser incluídos em estudo de manejo varietal em outras regiões paulistas.
\end{abstract}

Termos de indexação: cana-de-açúcar, Saccharum spp., melhoramento genético, hibridação, avaliação de clones.

( $\left.{ }^{1}\right)$ Trabalho recebido para publicação em 10 de fevereiro e aceito em 26 de julho de 1995.

$\left(^{2}\right)$ Estação Experimental de Jaú, Instituto Agronômico de Campinas (IAC), Caixa Postal 66, 17201-970 Jaú (SP).

$\left(^{3}\right)$ Seção de Cana-de-Açúcar, IAC.

(4) Estação Experimental de Ribeirão Preto, IAC.

(5) Divisão de Estações Experimentais, IAC.

(6) Com bolsa de pesquisa do CNPq. 


\title{
ABSTRACT \\ SUGARCANE BREEDING: X. EVALUATION OF CLONES OBTAINED \\ BY HIBRIDIZATION IN 1981 AND SELECTED IN JAÚ REGION, STATE OF SÃO PAULO, BRAZIL
}

\begin{abstract}
Seven sugarcane clones obtained from crosses made in 1981 in Camamu, State of Bahia, were evaluated in a trial in Jaú region. In the trial, started in February, 1988 , it was used a randomized complete block design with six replications. Statistical analysis for cane and sugar yield ( $t / h a$ ) and sugar content (pol\% cane) were performed with five harvest means. It was presented evaluation data of number of stalks, fibre\% cane and flowering intensity. According to these characteristics studied comparatively to five varieties used as control, IAC67-112, NA56-79, SP70-1143, SP71-1406 and PO77-102, the clones IAC81-3041 and IAC81-3049 showed be promising and in condition to be included in varietal management studies for others regions of the State of São Paulo, Brazil.
\end{abstract}

Index terms: sugarcane, Saccharum spp., breeding, hibridization, clone evaluation.

\section{INTRODUÇÃo}

O Instituto Agronômico, de Campinas, em um trabalho contínuo, vem-se dedicando, há muito tempo, ao melhoramento genético da cana-de-açúcar, visando obter, anualmente, novas variedades (Aguirre Junior, 1936; Segalla \& Alvarez, 1964, 1968).

A criação de novas variedades de cana atenderia plenamente a seus fins se produzisse genótipos com potencial agroindustrial semelhante ao das variedades em cultivo. A necessidade de novas variedades deve-se à "vida" relativamente curta dos cultivares mais utilizados (Brieger, 1978), em função do "declínio varietal" decorrente, sobretudo, da disseminação de doenças durante a propagação vegetativa. Dessa forma, recomenda-se a diversificação no plantio de variedades, estabelecendo-se grupos de cultivares com características semelhantes quanto ao uso agroindustrial.

O melhoramento da cana-de-açúcar inicia-se com a obtenção de sementes pela hibridação, mediante a qual se procura ampla variabilidade genética. Isolam-se os fenótipos desejados por meio de sucessivas seleções, levando-os, a seguir, à experimentação em ensaios regionais para a indicação de novas variedades de uso comercial.
Neste trabalho, apresentam-se os resultados do ensaio da região de Jaú (SP), com clones provenientes de hibridações executadas em 1981.

\section{MATERIAL E MÉTODOS}

Os clones utilizados neste trabalho originaram-se de hibridações realizadas em 1981, na Estação Experimental de Camamu (BA) da Cooperativa dos Produtores de Cana, Açúcar e Álcool do Estado de São Paulo (COPERSUCAR). Em uma primeira fase, selecionaram-se plantas individuais, por critério visual, identificando-se touceiras, com um mínimo de perfilhos (normalmente seis) e altura e diâmetro do colmo que evidenciassem o genótipo em relação ao comportamento médio da população oriunda de "seedlings".

Posteriormente, realizaram-se multiplicações vegetativas, colocando o clone em igualdade de condições com padrões comerciais, acompanhando-se a evolução fenológica por avaliação visual e emprego de escala conceitual para as características de altura, perfilhamento, germinação e brotação de soqueira. Para eleição dos clones a ser incluídos nesse ensaio de competição, efetuaram-se, ainda, duas análises tecnológicas, abrangendo um período precoce (maio) e o meio da safra (início de 
agosto). Dessa forma, instalou-se um ensaio definitivo de fevereiro de 1988 a outubro de 1993, em latossolo roxo distrófico na Usina da Barra, em Barra Bonita (SP).

Os dados de precipitação pluvial registrados no período de fevereiro de 1988 a outubro de 1993, no posto meteorológico da Estação Experimental de Jaú, encontram-se na figura 1.

O delineamento experimental foi em blocos ao acaso com seis repetições, comparando-se sete clones do programa de melhoramento de cana IAC em relação às variedades: IAC67-112, NA56-79, SP70-1143, SP71-1406 e PO77-102.

Os clones IAC avaliados e seus respectivos parentais foram os seguintes:

\section{Clones}

\section{Parentais}

IAC $81-3041 \ldots \ldots \ldots \ldots \ldots$ TUC56-19 $x$ ?

IAC $81-3049 \ldots \ldots \ldots \ldots \ldots$ TUC56-19 $\mathrm{x}$ ?

IAC $81-3072 \ldots \ldots \ldots \ldots \ldots$ TUC56-19 $x$ ?

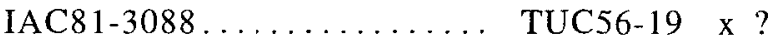

IAC $81-3095 \ldots \ldots \ldots \ldots \ldots$ IANE55-33 $x$ ?

IAC81-3129........... Co419 x ?

IAC81-3137........... Co419 $\mathrm{x}$ ?

As parcelas constituíram-se de 5 linhas de 8 $\mathrm{m}$, espaçadas de $1,40 \mathrm{~m}$, com área útil de $56 \mathrm{~m}^{2}$. A adubação utilizada em cana-planta foi de 400 $\mathrm{kg} / \mathrm{ha}$ da fórmula 04-20-20, e de $60 \mathrm{~kg}$ de $\mathrm{N}$ e $70 \mathrm{~kg}$ de $\mathrm{K}_{2} \mathrm{O} \mathrm{em}$ cobertura por hectare. Nas soqueiras, empregaram-se $500 \mathrm{~kg} / \mathrm{ha}$ de fórmula 20 -00-32, por ocasião do cultivo da cana.
O quadro 1 apresenta as datas de plantio, colheita e duração de cada ciclo (em meses).

Por ocasião das colheitas $\left(1 .^{\circ}\right.$ ao $5 .^{\circ}$ corte), amostraram-se dez colmos, seguidos na linha, em cada parcela, para análise tecnológica (Tanimoto, 1964), a fim de obter dados de pol\% cana e fibra\% cana.

Ao lado do ensaio, instalou-se um campo de multiplicação com três sulcos de $8 \mathrm{~m}$, em cada tratamento, para determinação da curva de maturação. As amostragens foram realizadas em cana-planta (1989), de abril a setembro e, na 1. ${ }^{2}$ soca (1990), entre maio e outubro, com intervalos de 30 dias, coletando-se, de cada tratamento, três amostras de dez canas, seguidas na linha e analisadas pelo método da prensa hidráulica (Tanimoto 1964).

Realizaram-se análises estatísticas dos dados de número de colmos; da produtividade de cana, calculada pela tonelada de cana por hectare (TCH); do teor de açúcar (pol\% cana); da produtividade de açúcar, estimada pela tonelada de pol por hectare (TPH), e fibra\% cana, utilizando-se o teste de Tukey a $5 \%$ para as comparações de médias.

Obteve-se a queda de produção $(\mathrm{Q} \%)$ do $1 .^{\circ}$ ao $5 .^{\circ}$ corte, empregando-se a expressão adaptada de Alvarez et al. (1987):

$$
\mathrm{Qx} \%=[(\mathrm{P} 1-\mathrm{Px}) / \mathrm{P} 1] \times 100
$$

onde:

Px representa a produção do corte considerado e P1, a produção de cana-planta.

Quadro 1. Datas de plantio, colheita e duração dos ciclos (em meses) do ensaio da série 81

\begin{tabular}{|c|c|c|c|c|c|c|c|c|c|c|}
\hline \multirow{3}{*}{$\begin{array}{c}\text { Plantio } \\
1988\end{array}$} & \multicolumn{5}{|c|}{ Cortes } & \multicolumn{5}{|c|}{ Ciclos } \\
\hline & $1 .{ }^{\circ}$ & $2 .^{\circ}$ & $3 .^{\circ}$ & $4 .^{\circ}$ & $5 .^{\circ}$ & $1 .^{\circ}$ & $2 .^{\circ}$ & $3 .^{\circ}$ & $4 .^{\circ}$ & $5 .^{\circ}$ \\
\hline & 1989 & 1990 & 1991 & 1992 & 1993 & & & Ieses & & \\
\hline $10 / 2$ & $9 / 8$ & $5 / 9$ & $9 / 9$ & $16 / 10$ & $16 / 10$ & 18,0 & 12,7 & 12,1 & 13,4 & 12,0 \\
\hline
\end{tabular}




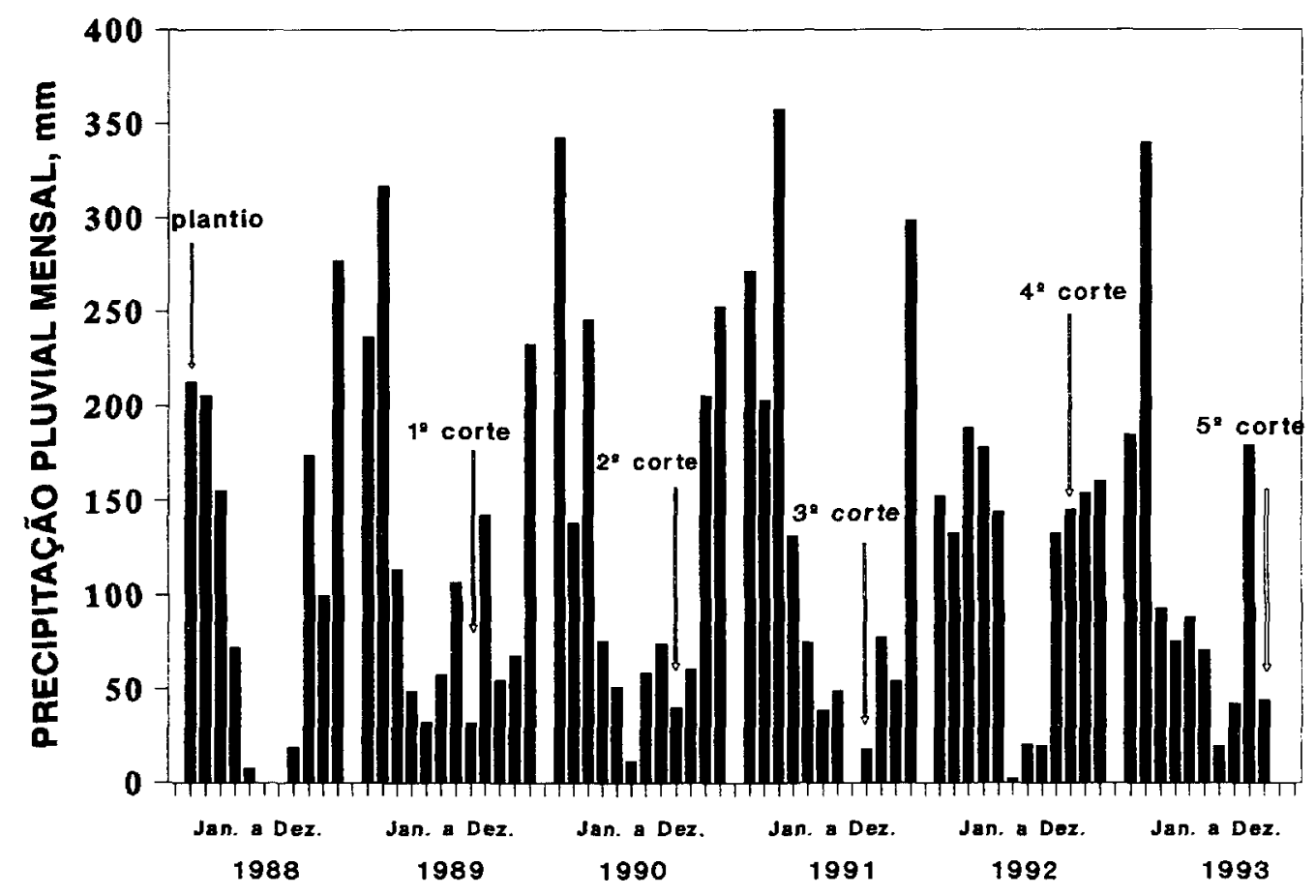

Figura 1. Dados de precipitação pluvial (total mensal em $\mathrm{mm}$ ) registrados no período de fevereiro/88-outubro/93, em Jaú (SP).

\section{RESULTADOS E DISCUSSÃO}

O quadro 2 mostra os valores médios de cinco colheitas para produtividade de cana, teor de sacarose, produtividade de açúcar, número de colmos e fibra\% cana.

Com relação à produtividade de cana, o padrão IAC67-112 apresentou o maior valor, embora não diferisse, estatisticamente, dos clones IAC81-3088, IAC81-3049, IAC81-3095, IAC81-3072 e IAC81-3041 e dos padrões SP70-1143, SP71-1406 e NA56-79.

Quanto ao teor de sacarose, o clone IAC81-3129 revelou o maior valor, mas, em contrapartida, a menor produtividade agrícola entre os tratamentos; mesmo não diferindo estatisticamente deste último, quanto ao pol\% cana, pôde-se conferir destaque ao IAC81-3041, já que esses dois clones se equivaleram aos padrões IAC67-112 e PO77-102 e superaram os demais.

Na produtividade de açúcar, resultado do produto da tonelada de cana pelo teor de sacarose, o padrão IAC67-112 diferenciou-se significativamente dos demais tratamentos. Em um segundo grupo, destacaram-se os clones IAC81-3049, IAC81-3041 e IAC81-3088, os quais se equipararam aos padrões SP70-1143, SP71-1406, NA56-79 e PO77-102.

Dentre os melhores tratamentos para produtividade agrícola, sobressaíram-se, quanto ao número de colmos, os clones IAC81-3095 e IAC81-3072, ambos equivalentes ao padrão SP70-1143. Num segundo grupo, os clones IAC81-3049 e IAC81-3137 igualaram-se estatisticamente aos padrões IAC67-112, SP71-1406, NA56-79 e PO77-102.

Quanto à fibra\% cana, os clones IAC81-3049 e IAC81-3088 equivaleram-se aos padrões SP70-1143 e SP7 1-1406, com teores médios de fibra.

Pelo quadro 3, verifica-se que, entre os melhores clones em produção de açúcar por área, o IAC81-3041 e o IAC81-3049, revelaram os menores índices de queda de produção de cana ao longo das cinco colheitas, equiparando-se aos padrões SP70-1143, NA56-79 e IAC67-112, além de um nível 
de florescimento inferior a $10 \%$. O clone IAC 81 -3088 , que se havia destacado em produtividade de cana e açúcar, manifestou índice de queda de produção e florescimento elevados, inviabilizando- se como variedade. Os demais clones apresentaram alto índice de queda de produção de cana, assim como o padrão SP71-1406.

O quadro 4 relaciona as cquações de segundo grau, que expressam as maturações dos genótipos em cana-planta e cana-soca.

O $\mathbf{x}$ admite os valores de 0 a 120 tanto para cana-planta como para cana-soca, referente às épocas de amostragens de pol\% cana após o início da safra; deve-se, portanto, substituí-lo pelo número de dias desejado, contando-se a partir de $27 / 4$ para cana-planta e $16 / 5$ para soca.
Compararam-se as curvas de maturação dos clones IAC81-3041 e IAC81-3049 às dos padrões NA56-79 e SP70-1143, que, na ocasião, estavam entre as variedades mais plantadas no Estado de São Paulo (Braga Junior, 1991) (Figuras 1 e 2).

Considerando que os últimos pontos da curva de maturação foram tomados em agosto, a equação quadrática do tipo $\mathrm{y}=\mathrm{a}+\mathrm{bx}+\mathrm{cx}^{2}$ talvez não seja a mais adequada para o ajuste dos dados na curva (a mais adequada, nesse caso, seria a equação linear), mesmo assim possibilita a interpretação do teor de sacarose inicial (valor a), ou seja, da precocidade, e da inclinação da curva do início ao meio de safra (valor b, dado pela razão do valor da ordenada (y) pela abscissa $(\mathbf{x})$. O ponto de máxima, calculado a partir da derivada dessa equação, não deve ser aplicado nesse caso.

Quadro 2. Médias de cinco cortes (1989/93) de produtividade de cana e açúcar, teor de sacarose, número de colmos, fibra\% cana e resumo da análise da variância do experimento com clones IAC da série 81 , instalado na Usina da Barra, na região de Jaú (SP)

\begin{tabular}{lccccc}
\hline Tratamentos & $\begin{array}{c}\text { Produtividade } \\
\text { de cana }\end{array}$ & $\begin{array}{c}\text { Teor } \\
\text { de sacarose }\end{array}$ & $\begin{array}{c}\text { Produtividade } \\
\text { de açúcar }\end{array}$ & $\begin{array}{c}\text { Número } \\
\text { de colmos }\end{array}$ & Fibra \\
\hline IAC-67-112 & TCH & Pol\% Cana & t de Pol/ha & Linha de $10 \mathrm{~m}$ & $\%$ \\
SP70-1143 & $110,5 \mathrm{a}$ & $15,0 \mathrm{ab}$ & $16,5 \mathrm{a}$ & $92,1 \mathrm{bc}$ & $11,8 \mathrm{~cd}$ \\
SP71-1406 & $103,2 \mathrm{a}-\mathrm{c}$ & $14,4 \mathrm{~cd}$ & $14,9 \mathrm{~b}$ & $110,2 \mathrm{a}$ & $13,7 \mathrm{ab}$ \\
IAC81-3049 & $101,3 \mathrm{a}-\mathrm{c}$ & $14,6 \mathrm{~b}-\mathrm{d}$ & $14,8 \mathrm{~b}$ & $92,1 \mathrm{bc}$ & $13,7 \mathrm{ab}$ \\
IAC81-3041 & $102,9 \mathrm{a}-\mathrm{c}$ & $14,3 \mathrm{~d}$ & $14,7 \mathrm{bc}$ & $92,9 \mathrm{bc}$ & $14,6 \mathrm{a}$ \\
IAC81-3088 & $95,7 \mathrm{a}-\mathrm{c}$ & $15,2 \mathrm{a}$ & $14,6 \mathrm{bc}$ & $84,5 \mathrm{~cd}$ & $11,7 \mathrm{~d}$ \\
PO77-102 & $104,9 \mathrm{ab}$ & $13,4 \mathrm{c}$ & $13,9 \mathrm{~b}-\mathrm{d}$ & $83,3 \mathrm{~cd}$ & $13,1 \mathrm{a}-\mathrm{d}$ \\
NA56-79 & $91,9 \mathrm{bc}$ & $15,0 \mathrm{ab}$ & $13,9 \mathrm{~b}-\mathrm{d}$ & $92,6 \mathrm{bc}$ & $13,3 \mathrm{a}-\mathrm{c}$ \\
IAC81-3072 & $95,1 \mathrm{a}-\mathrm{c}$ & $14,9 \mathrm{~d}$ & $13,7 \mathrm{~b}-\mathrm{d}$ & $90,5 \mathrm{bc}$ & $12,5 \mathrm{~b}-\mathrm{d}$ \\
IAC81-3137 & $96,8 \mathrm{a}-\mathrm{c}$ & $14,1 \mathrm{~d}$ & $13,7 \mathrm{~b}-\mathrm{d}$ & $98,8 \mathrm{ab}$ & $12,4 \mathrm{~b}-\mathrm{d}$ \\
IAC81-3129 & $92,4 \mathrm{bc}$ & $14,5 \mathrm{~cd}$ & $13,4 \mathrm{c}-\mathrm{e}$ & $85,5 \mathrm{~b}-\mathrm{d}$ & $12,4 \mathrm{~b}-\mathrm{d}$ \\
IAC81-3095 & $87,7 \mathrm{c}$ & $15,2 \mathrm{a}$ & $13,2 \mathrm{de}$ & $75,7 \mathrm{~d}$ & $11,6 \mathrm{~d}$ \\
\hline F & $97,0 \mathrm{a}-\mathrm{c}$ & $12,6 \mathrm{f}$ & $12,2 \mathrm{e}$ & $110,4 \mathrm{a}$ & $12,8 \mathrm{~b}-\mathrm{d}$ \\
\hline d.m.8. (Tukey $5 \%)$ & $3,7 * *$ & $50,4 * *$ & $15,9 * *$ & $12,2 * *$ & $8,1 * *$ \\
CV (\%) & 16,1 & 0,5 & 1,3 & 14,2 & 1,6 \\
\hline
\end{tabular}

**:Significativo ao nível de $1 \%$. 


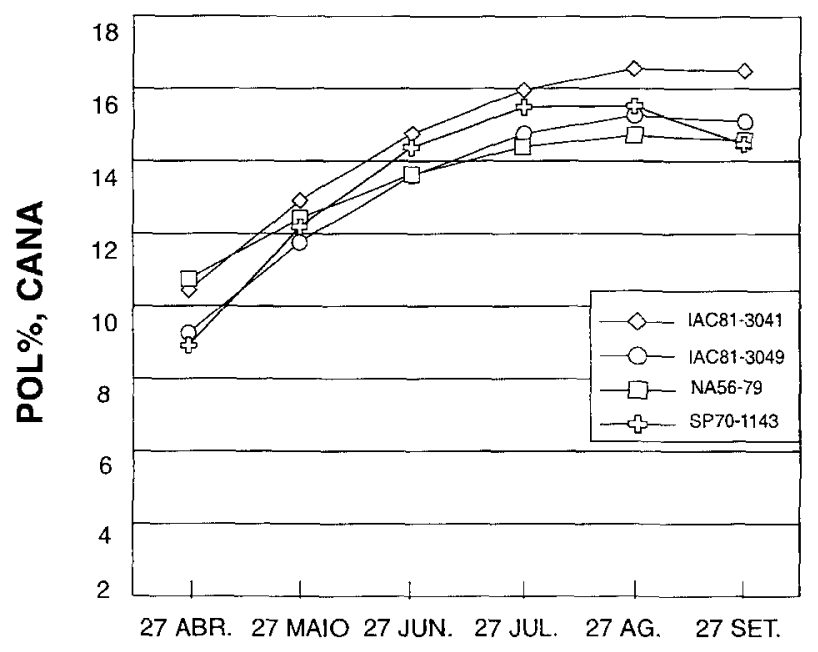

ÉPOCAS DE AMOSTRAGEM

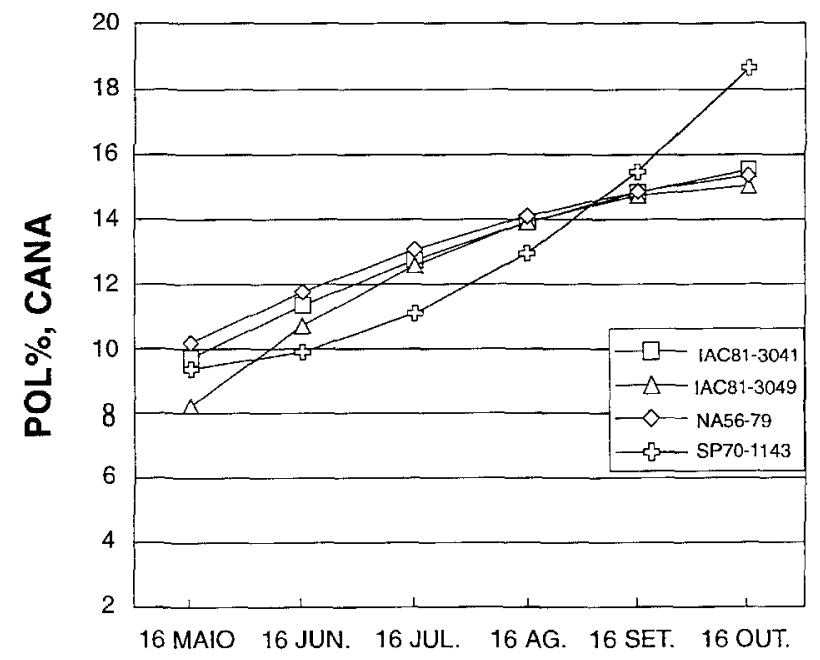

ÉPOCAS DE AMOSTRAGEM
Figura 2. Curvas de maturação de clones IAC e testemunhas na cana-planta, em ensaio realizado na Usina da Barra, na região de Jaú (SP)
Figura 3. Curvas de maturação de clones IAC e testemunhas na cana-soca, em ensaio realizado na Usina da Barra, na região de Jaú (SP)

Quadro 3. Valores de índice de queda de produção da $2 .^{a}$ à $5 .^{a}$ colheita (Q\%) e de intensidade de florescimento do experimento com clones IAC, da série 81, instalado na Usina da Barra, na região de Jaú (SP)

\begin{tabular}{|c|c|c|c|c|c|}
\hline \multirow{2}{*}{ Tratamentos } & \multicolumn{4}{|c|}{ Índices de queda } & \multirow{2}{*}{ Florescimento $\left({ }^{1}\right)$} \\
\hline & $\mathrm{Q} 2$ & Q3 & Q4 & Q5 & \\
\hline IAC $67-112$ & 21,4 & 43,2 & 24,3 & 16,9 & 0 \\
\hline NA56-79 & 24,6 & 18,0 & 23,9 & 44,9 & 2 \\
\hline PO77-102 & 35,9 & 26,0 & 32,5 & 42,8 & 2 \\
\hline SP70-1143 & 20,5 & 28,6 & 20,8 & 31,7 & 2 \\
\hline SP71-1406 & 39,2 & 43,0 & 32,0 & 49,2 & 0 \\
\hline IAC $81-3041$ & 19,2 & 31,1 & 21,8 & 33,3 & 1 \\
\hline IAC81-3049 & 24,1 & 41,2 & 27,3 & 22,9 & 1 \\
\hline IAC81-3072 & 22,7 & 32,6 & 26,9 & 35,2 & 1 \\
\hline IAC $81-3088$ & 24,8 & 37,5 & 30,3 & 40,2 & 2 \\
\hline IAC81-3095 & 31,6 & 39,3 & 36,0 & 49,2 & 2 \\
\hline IAC 81-3129 & 23,1 & 37,4 & 27,0 & 32,4 & 0 \\
\hline IAC $81-3137$ & 35,2 & 43,3 & 40,7 & 40,7 & 1 \\
\hline
\end{tabular}

(1) Escala de 0 a 3 pontos: $0=$ Sem florescimento; $1=<10 \%$ de florescimento; $2=10-20 \%$ de florescimento; $3=>20 \%$ de florescimento. 


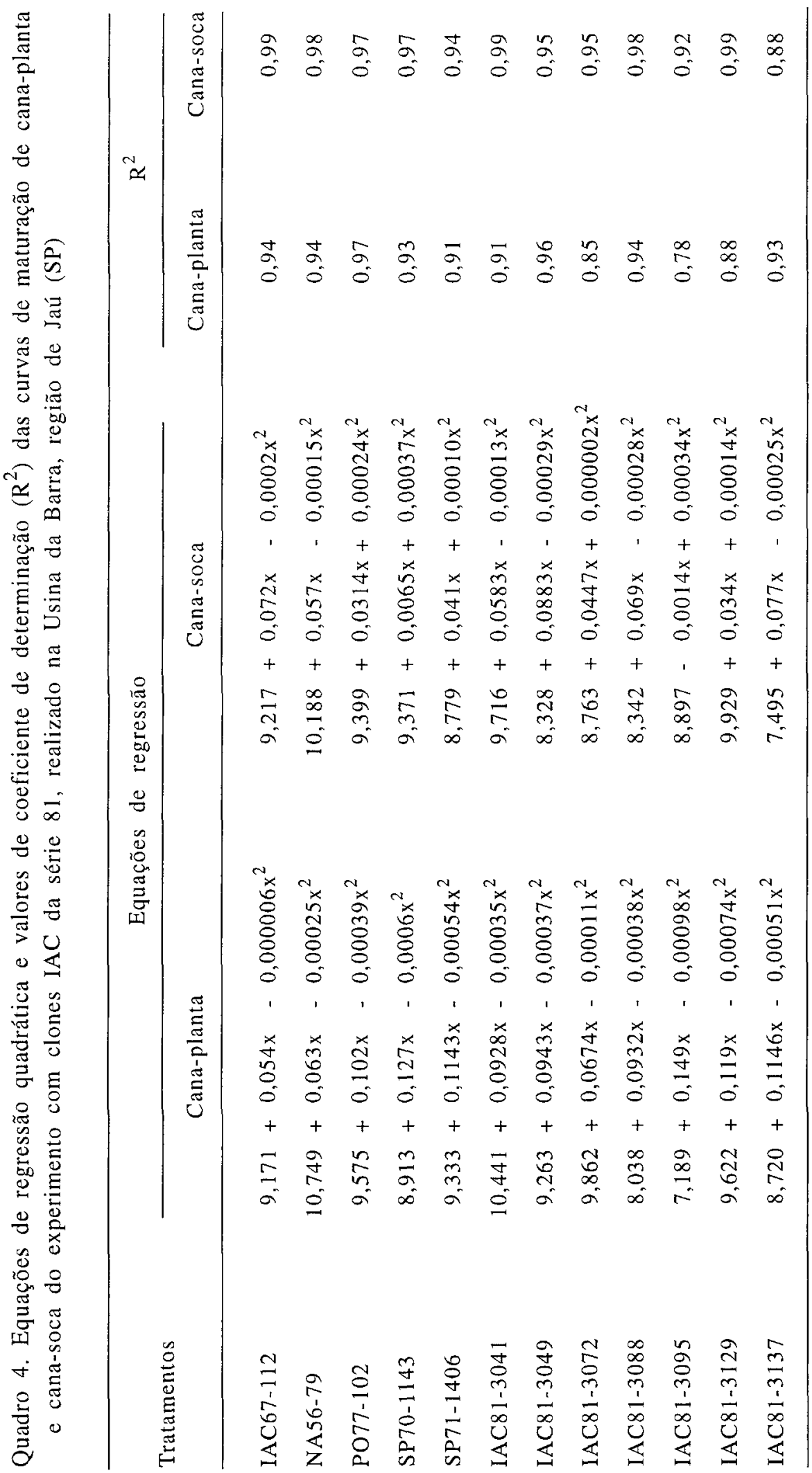


Em uma equação de $2 .^{\circ}$ grau, o componente bx vai perdendo importância à medida que aumentam os valores de $\mathbf{x}$. Nesse momento, o componente $\mathbf{c x}^{2}$ vai adquirindo valores cada vez maiores e ganhando importância na determinação do y. Como isso ocorre, neste caso, com o aumento dos números de dias após a safra, pode-se dizer que $\mathbf{c x}^{2}$ é o principal indicador da característica de maturação tardia de uma variedade.

Em cana-planta, o clone IAC81-3041, equivalente ao padrão NA56-79, no início da safra, apresentou-se, porém, a partir da segunda amostragem, superior em pol\% cana, mantendo-se, assim, até o final.

Em relação ao padrão SP70-1143, o IAC81-3041 sempre se mostrou superior desde o início da safra. Tal clone exibiu alto teor de sacarose durante o período, caracterizando-se como de longo período útil de industrialização (PUI). O clone IAC81-3049, cujos níveis de pol\% cana, se assemelharam aos da SP70-1143 e superaram os da NA56-79, a partir de julho, pode ser utilizado a partir do meio da safra (junho/julho).

Na maturação da cana-soca, o clone IAC81-3041 repetiu o comportamento verificado na cana-planta, quanto à precocidade e teor de sacarose, equiparando-se ao padrão NA56-79; nesse caso, porém, teve um PUI mais curto, e, a partir de setembro, a variedade-padrão SP70-1143 apresentou melhores teores para colheita. O clone IAC81-3049 mostrou comportamento semelhante ao da cana-planta até

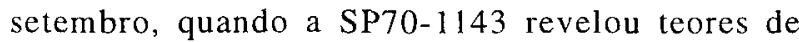
sacarose mais elevados. Tanto o IAC81-3041 como o IAC81-3049 apresentaram baixos índices de florescimento (Quadro 3), e o padrão SP70-1143, ínđices maiores. Sendo assim, o manejo de colheita dos clones pode-se estender por mais tempo na safra, enquanto o da SP70-1143 tem de ser reduzido.

\section{CONCLUSÕES}

1. Entre os genótipos avaliados, os clones IAC81-3041 e IAC81-3049 apresentaram características agroindustriais satisfatórias, quando cultivados em solos de fertilidade média e colhidos, respectivamente, a partir do início e meados da safra, na região de Jaú.

2. Os clones IAC81-3041 e IAC81-3049 revelaram-se apropriados para ser incluídos em ensaios de manejo varietal nas demais regiões paulistas.

\section{AGRADECIMENTOS}

À Cooperativa dos Produtores de Cana, Açúcar e Álcool do Estado de São Paulo (COPERSUCAR), por permitir a utilização de seus campos de cruzamentos, na Estação Experimental de Camamu (BA).

À Usina Da Barra S.A., por permitir a instalação do ensaio, e ao seu corpo técnico de experimentação, pelo apoio na instalação, desenvolvimento e colheita do ensaio.

\section{REFERÊNCIAS BIBLIOGRÁFICAS}

AGUIRRE JUNIOR, J.M. Criação de novas variedades de cana no Estado de São Paulo. Campinas, Instituto Agronômico, 1936. 64p. (Boletim técnico, 34)

ALVAREZ, R.; SEGALLA, A.L.; LANDELL, M.G.A.; SILVAROLLA, M.B. \& GODOY JUNIOR, G. Melhoramento genético da cana-de-açúcar: avaliação de clones provenientes de hibridações efetuadas em 1965. Bragantia, Campinas, 46(1):121-126, 1987.

BRAGA JUNIOR, R.L.C. Variedades mais cultivadas pelos produtores cooperados à Copersucar em 1990 In: SEMINÁRIO DE TECNOLOGIA AGRONÔMICA, 5., Piracicaba, 1991. Anais. Piracicaba, Copersucar, 1991. p.71-77.

BRIEGER, F. Situação do melhoramento da cana-deaçúcar no Estado de São Paulo. In: 50 anos da Estação Experimental de Piracicaba. Campinas, Instituto Agronômico, 1978. 82p.

SEGALLA, A.L. \& ALVAREZ, R. Melhoramento da cana-de-açúcar: I. Experiências com "seedlings" obtidos em 1947, 1948 e 1949. Bragantia, Campinas, 23:187-223, 1964.

SEGALLA, A.L. \& ALVAREZ, R. Contribuição ao desenvolvimento da cultura de cana-de-açúcar em Sāo Paulo. O Agronômico, Campinas, 20(5/6):1-35, 1968.

TANIMOTO, T. The press method of cane analysis. $\mathrm{Ha}$ waiian Planter's Record, Honolulu, 57:133-150, 1964. 